\title{
A influência da idade e do genótipo sobre os níveis de cloreto do suor em uma população pediátrica com fibrose cística no estado da Bahia
}

\author{
The influence of age and genotype on sweat chloride levels in a pediatric population \\ with cystic fibrosis in the state of Bahia
}

\begin{abstract}
Adson Santana de Jesus ${ }^{1}$ Edna Lúcia Souza2*
${ }^{1}$ Licenciado em Ciências Biológicas. Mestrando do Programa de Pós-Graduação em Processos Interativos dos Órgãos e Sistemas, Instituto de Ciência da Saúde, Universidade Federal da Bahia. ${ }^{2}$ Doutora em Medicina e Saúde, Professora Associada do Departamento de Pediatria da Faculdade de Medicina da Bahia, Universidade Federal da Bahia.
\end{abstract}

\begin{abstract}
Resumo
Introdução: a fibrose cística é a doença autossômica recessiva mais comum em populações caucasianas e a sua etiologia está associada a variantes patogênicas no gene CFTR. $O$ teste do suor é considerado o padrão ouro para o diagnóstico dessa enfermidade. Estudos apontam que o genótipo do CFTR e a idade dos indivíduos influenciam as concentrações de cloreto no suor. Objetivos: pesquisar a correlação entre os níveis de cloreto no teste do suor e a idade ao diagnóstico de indivíduos com fibrose cística e comparar as concentrações iônicas do cloreto entre os sexos, diferentes faixas etárias e três grupos diversos de genótipos do CFTR. Metodologia: realizou-se um estudo de corte transversal, incluindo sujeitos de 0 a 20 anos, com diagnóstico confirmado de fibrose cística. Os indivíduos selecionados foram agrupados de acordo com as variáveis analisadas. Calcularam-se os valores descritivos das concentrações de íons cloreto de cada grupo. Utilizou-se o teste de Spearman para a análise da correlação entre a idade ao diagnóstico e os níveis de cloreto no suor. Resultados: 64 indivíduos foram incluídos no estudo, sendo 51,56\% do sexo masculino. A mediana (Min - Max) da idade ao diagnóstico foi de 7 meses (1-206). Não foi observada correlação entre a idade dos indivíduos ao diagnóstico e os níveis de cloreto no suor. As concentrações medianas de cloreto foram maiores nos escolares (106 mEq/l), no sexo feminino (102 mEq/l) e nos heterozigotos F508del/Classe I a III (108 mEq/l); e menores nos adolescentes (100 mEq/l) e nos heterozigotos F508del/Classes IV a VI (77 mEq/I). Conclusão: os níveis de cloreto no suor não apresentaram correlação com a idade dos indivíduos ao diagnóstico. A variação considerável dos níveis iônicos entre os grupos de diferentes genótipos corrobora que o teste do suor é um bom preditor da avaliação funcional do canal CFTR.

Palavras-chave: Mucoviscidose. Teste do suor. Mutações. CFTR.
\end{abstract}

\begin{abstract}
Introduction: cystic fibrosis is the most common autosomal recessive disorder in Caucasian populations and its etiology is associated with pathogenic variants in the CFTR gene. The sweat test is considered the gold standard for the diagnosis of the disease. Some studies suggest that CFTR genotype and age affect sweat chloride concentrations. Objectives: to investigate the correlation between sweat chloride levels and age at diagnosis of individuals with cystic fibrosis and to compare ionic chloride concentrations among sexes, different age groups and three distinct groups of CFTR genotypes. Methodology: a cross-sectional study was conducted, which included CF subjects from 0 to 20 years of age. The selected individuals were clustered on the variables in analysis. The description values for chloride ion concentrations in each group were calculated. The Spearman's test was used to analyze the correlation between the age at diagnosis and sweat chloride levels. Results: 64 individuals were included, 51,56\% male. The median (Min - Max) age at diagnosis was 7 months (1-206). There was no correlation between the age at diagnosis and sweat chloride levels. The median of the chloride concentrations were higher for schoolchildren (106 mEq/l), females (102 mEq/l) and heterozygous F508del/Classes I to III (108 mEq/I), and reached the lowest values for teenagers (100 mEq/I) and heterozygous F508del/Classes IV to VI (77 mEq/I). Conclusion: sweat chloride levels did not correlate with the age of individuals at diagnosis. The substantial variation of ionic levels among groups of distinct genotypes corroborates that the sweat test is a good predictor for functional assessment of the CFTR channel. Keywords: Mucoviscidosis. Sweat test. Mutations. CFTR.
\end{abstract}

\section{INTRODUÇÃO}

A fibrose cística ( $\mathrm{FC}$ ) é a doença genética autossômica recessiva mais comum em populações de origem caucasia-

Correspondente/Corresponding: *Edna Lúcia Souza - Tel: - E-mail: souza.ednalucia@gmail.com na (VIDIGAL et al. 2008). A etiologia se associa a variantes patogênicas no gene Cystic Fibrosis Transmembrane Conductance Regulator (CFTR), o qual codifica um canal homônimo de íons cloreto e de bicarbonato, localizado na membrana de células epiteliais (FIRMIDA; MARQUES; DA COSTA, 2011; PEREIRA et al. 2019).

Segundo o Cystic Fibrosis Mutation Database (2020), mais de 2100 variantes já foram descritas para o gene 
CFTR, sendo que 360 delas são consideradas patogênicas (CYSTIC FIBROSIS FOUNDATION, 2020). As variantes patogênicas no gene supracitado são classificadas em seis tipos, de acordo com os efeitos que causam sobre a síntese ou a função da proteína CFTR e, consequentemente, sobre as manifestações clínicas da doença (DE BOECK; AMARAL, 2016; MARSON, 2018). Os indivíduos com variantes das classes I a III, com ausência de função da CFTR, geralmente, apresentam concentrações mais elevadas de íons cloreto no suor e insuficiência pancreática exócrina (FIRMIDA; MARQUES; DA COSTA, 2011; ELBORN, 2016). Neste sentido, as mutações das classes IV a VI associam-se com alguma função residual da CFTR, concentrações moderadas de cloreto no suor e suficiência do pâncreas exócrino (ELBORN, 2016).

O teste do suor consiste na medida dos níveis de cloreto ou de cloreto e de sódio no suor excretado e é considerado o padrão ouro para o diagnóstico da FC (ATHANAZIO et al. 2017). Os níveis desses íons no suor refletem a atividade da proteína CFTR e, dessa forma, podem ser considerados biomarcadores dessa funcionalidade (ESPEL et al. 2018). Sugere-se, também, que as concentrações do cloreto no suor sejam variáveis nas diferentes faixas etárias. Contudo, alguns estudos apresentaram resultados conflitantes sobre a esta relação, principalmente nos primeiros anos de vida (PARAD et al., 2005; TRAEGER et al., 2014; FARIA et al., 2017).

A variante patogênica F508del é a mais comum mundialmente e, geralmente, é associada à ausência de funcionalidade da proteína CFTR, sendo classificada na classe II. A ocorrência desta variante em homozigose ou em heterozigose composta com outras variantes patogênicas das classes I a III tem sido associada a altas concentrações de íons cloreto no suor (WILSCHANSKI et al., 1995; FARREL; KOSCIK, 1996; ESPEL et al. 2018). Por outro lado, a heterozigose composta entre a F508del e outras variantes das classes IV a VI determina níveis de cloreto no suor que, embora variáveis, são geralmente menores do que aqueles encontrados nos genótipos supracitados (WILSCHANSKI et al., 1995; FELDMANN et al., 2003; ESPEL et al., 2018).

Diante do exposto, os objetivos deste estudo foram pesquisar a correlação entre os níveis de cloreto no teste do suor e a idade ao diagnóstico de indivíduos com fibrose cística e comparar as concentrações iônicas do cloreto entre os sexos, diferentes faixas etárias e três grupos diversos de genótipos do CFTR.

\section{METODOLOGIA}

\section{Desenho do Estudo}

Trata-se de um estudo de corte transversal em uma população pediátrica acompanhada no Ambulatório Multiprofissional de Fibrose Cística do Hospital Universitário Professor Edgard Santos, da Universidade Federal da Bahia, a partir do ano de 2008.

\section{População}

Incluíram-se todos os indivíduos com idade entre $0 \mathrm{e}$ 20 anos, com diagnóstico de $\mathrm{FC}$ realizado através de dois resultados positivos do teste do suor (níveis de cloreto $\geq$ $60 \mathrm{mEq} / \mathrm{L}$ ) e/ou pela presença de duas variantes patogênicas do gene CFTR. Excluíram-se do estudo os indivíduos com teste do suor duvidoso (níveis de cloreto entre 30 e $59 \mathrm{mEq} / \mathrm{L}$ ) e com pelo menos uma mutação de significado incerto ou desconhecido.

\section{Protocolo de estudo}

Os indivíduos foram submetidos ao teste do suor para o diagnóstico da doença. Este procedimento foi realizado através do método de iontoforese por pilocarpina descrito por Gibson e Cooke (1959). Dois exames foram realizados e, em cada um deles, analisaram-se duas medidas das concentrações de cloreto para a confirmação diagnóstica. Além disto, a pesquisa de variantes patogênicas no gene CFTR foi realizada através da reação em cadeia da polimerase, associada à aplicação de enzimas de restrição (PCR-RFLP) ou por meio do sequenciamento de nova geração.

Os dados clínicos foram coletados no período de outubro a novembro de 2020, a partir da ficha clínica preenchida com os registros dos prontuários médicos. Estudaram- se as seguintes variáveis: idade ao diagnóstico, sexo, os níveis de cloreto no suor (sendo adotado o maior valor encontrado para cada paciente) e o genótipo.

\section{Aspectos Éticos}

O estudo foi aprovado pelo Comitê de Ética em Pesquisa do Hospital Universitário Professor Edgard Santos, pelo Parecer n. 4.321.551. Os indivíduos ou os seus responsáveis legais assinaram o termo de consentimento livre e esclarecido e, quando pertinente, as crianças assinaram o termo de assentimento.

\section{Análise estatística}

Os dados obtidos foram registrados e armazenados no software Microsoft Excel 365. O software R-Studio 1.3.1093 foi utilizado para análise de correlação. A estatística descritiva consistiu nos cálculos das frequências simples e relativas das variáveis estudadas. As crianças foram classificadas nas seguintes faixas etárias: lactentes ( 0 a 23 meses e 29 dias), pré-escolares ( 24 a 71 meses e 29 dias), escolares ( 72 meses a 119 meses e 29 dias) e adolescentes (120 a 227 meses e 29 dias) (SOCIEDADE BRASILEIRA DE PEDIATRIA, 2014).

As crianças foram categorizadas em três grupos, de acordo com seus genótipos: G1- homozigotos para a variante patogênica F508del; G2- heterozigotos compostos para a mutação F508del e uma variante das classes I a III; e G3- heterozigotos compostos para a mutação F508del e uma variante das classes IV a VI. Os indivíduos que não apresentavam a mutação F508del ou a apresentavam em heterozigose composta com outras variantes, cujas classes 
funcionais ainda não foram definidas, foram excluídos desta análise.

O teste de correlação de Spearman foi realizado para a análise da correlação entre os níveis de cloreto no suor e a idade ao diagnóstico. Os valores medianos do teste do suor foram comparados entre os sexos, as faixas etárias e os diferentes grupos de genótipo do CFTR.

\section{RESULTADOS}

Incluíram-se 64 crianças, sendo excluído um indivíduo por apresentar teste do suor duvidoso e não ter genótipo definido; 33 (51,56\%) foram do sexo masculino, mediana de idade (Min- Max) ao diagnóstico de 7 meses (1-206).

As frequências de indivíduos e a mediana dos níveis de cloreto no suor por faixa etária estão descritas na Tabela 1. A variação entre essas medianas nesses grupos está representada no Gráfico 1. As medianas (Min-Max) de idade (em meses), ao diagnóstico, foram de 4 (1-18), 49,5 (32-68), 94,5 (90-106) e 171 (136-206) para os lactentes, pré-escolares, escolares e adolescentes, respectivamente.

Tabela 1 - Frequências de indivíduos e a mediana dos valores das concentrações dos íons $\mathrm{Cl}$ - no suor (em $\mathrm{mEq} / \mathrm{l}$ ) de acordo com cada faixa etária ao diagnóstico da doença $(N=64)$

\begin{tabular}{lcc}
\hline Faixas etárias & \multicolumn{1}{c}{$\mathbf{N}(\%)$} & Valor mediano (I.I.Q) $^{\mathbf{a}}$ \\
\hline Lactentes & $41(64,06 \%)$ & $102(84-109)$ \\
Pré-escolares & $10(15,63 \%)$ & $101(84,5-112,25)$ \\
Escolares & $4(6,25 \%)$ & $106(90,75-114)$ \\
Adolescentes & $9(14,06 \%)$ & $100(80-106)$
\end{tabular}

Fonte: Dados da pesquisa.

Legenda a: I.I.Q-Intervalo interquartílico

Gráfico 1-Comparação das medianas e variações interquartílicas das concentrações dos íons $\mathrm{Cl}$ - no suor (em $\mathrm{mEq} / \mathrm{L}$ ) entre as faixas etárias da idade ao diagnóstico da doença.

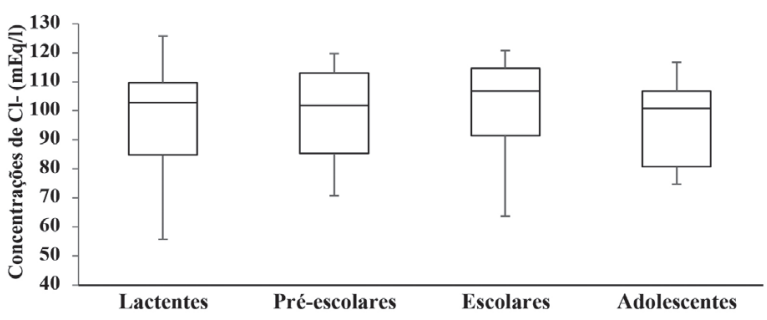

Fonte: Dados da pesquisa.

Não houve correlação entre as concentrações de cloreto no suor e a idade das crianças ao diagnóstico $(p=0,10)$ (Gráfico 2).
Gráfico 2 - Dispersão dos valores das concentrações de cloreto detectadas no teste do suor de acordo com a idade ao diagnóstico (em meses). Não houve correlação entre estas variáveis.

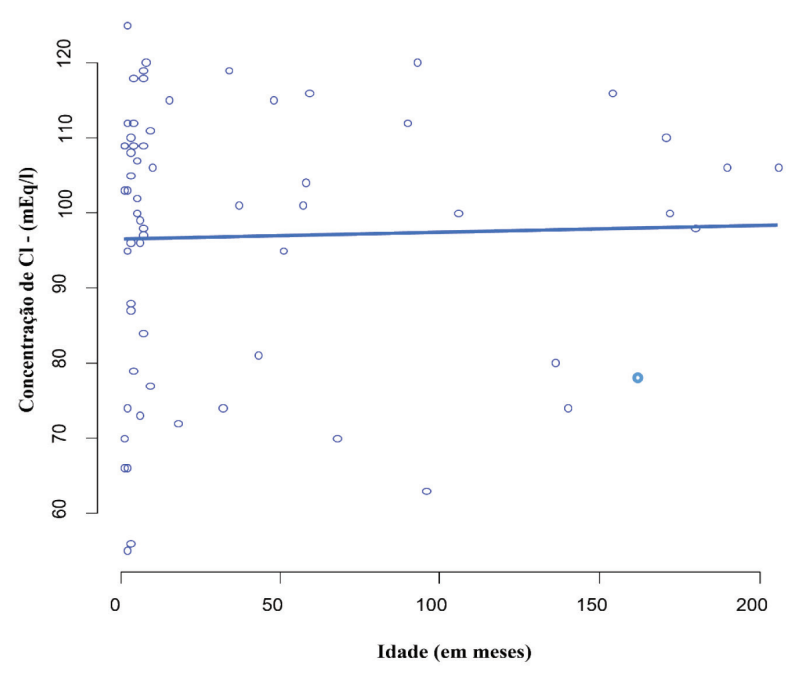

Fonte: Dados da pesquisa.

As frequências dos indivíduos e a mediana dos níveis de cloreto por sexo estão descritas na Tabela 2.

Tabela 2 - Frequências de indivíduos e a mediana dos valores das concentrações dos íons $\mathrm{Cl}$ - no suor (em $\mathrm{mEq} / \mathrm{l}$ ) para os sexos masculino e feminino $(N=64)$.

\begin{tabular}{lcc}
\hline \multicolumn{1}{c}{ Sexo } & $\mathbf{N}(\%)$ & Valor mediano (I.I.Q) $^{\text {a }}$ \\
\hline Masculino & $33(51,56 \%)$ & $100(84-108)$ \\
Feminino & $31(48,44 \%)$ & $102(79-113,5)$ \\
\hline
\end{tabular}

Fonte: Dados da pesquisa.

Legenda: a: I.I.Q-Intervalo interquartílico.

Dentre os 64 sujeitos selecionados, 37 indivíduos foram agrupados de acordo com o genótipo e 27 foram excluídos por não apresentarem a variante F508del ou por apresentarem em heterozigose composta com mutações de classes não definidas. As frequências dos indivíduos em cada grupo, bem como a mediana dos níveis de cloreto, estão descritas na Tabela 3. A variação entre estas medianas está representada no Gráfico 3.

Tabela 3 - Frequências de indivíduos e a mediana dos valores das concentrações dos íons $\mathrm{Cl}$ - no suor (em $\mathrm{mEq} / \mathrm{l}$ ) para os três grupos de genótipos do CFTR (N=37).

\begin{tabular}{|c|c|c|}
\hline Grupo & $N(\%)$ & Valor mediano (I.I.Q) \\
\hline $\mathrm{G}^{\mathrm{a}}$ & $19(51,35 \%)$ & $107(97-111)$ \\
\hline $\mathrm{G} 2^{\mathrm{b}}$ & $15(40,54 \%)$ & $108(102-117)$ \\
\hline $\mathrm{G}^{\mathrm{c}}$ & $3(8,11 \%)$ & $77(71,5-86,5)$ \\
\hline
\end{tabular}

Fonte: Dados da pesquisa.

Legenda: a: G1: F508del/F508del. b: G2: F508del/Classes I a III. c: G3: F508del/Classes IV a VI. d: I.I.Q - Intervalo interquartílico. 
Gráfico 3-Comparação das medianas e variações interquartílicas das concentrações dos íons $\mathrm{Cl}$ - no suor (em $\mathrm{mEq} / \mathrm{L}$ ) entre os três grupos de genótipos do CFTR.

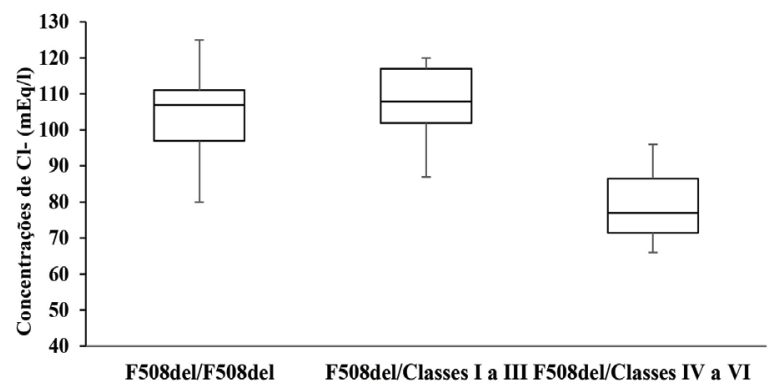

Fonte: Dados da pesquisa.

\section{DISCUSSÃO}

Os resultados observados evidenciaram medianas mais elevadas das concentrações de cloreto nos grupos 1 (F508del/F508del) e 2 (F508del/Classes I a III), quando comparados ao grupo 3 (F508del/Classes IV a VI). Estes resultados estão em consonância com outros estudos, onde os autores observaram que os níveis de cloreto de suor foram maiores nos indivíduos homozigotos para a mutação F508del e nos heterozigotos compostos com uma variante F508del e outra mutação das classes I a III, quando comparados àqueles que apresentavam uma mutação F508del em heterozigose composta com uma variante das classes IV a VI (WILSCHANSKI et al.,1995; FARREL; KOSCIK, 1996; ESPEL et al.,2018). Entretanto, neste estudo, apenas três crianças foram incluídas no grupo 3, o que pode dificultar a interpretação dos resultados.

Nos estudos de Feldmann et al. (2003) e Espel et al. (2018), as concentrações de cloreto em indivíduos heterozigotos compostos para as mutações F508del e $\mathrm{R} 117 \mathrm{H}$ (uma variante de classe IV) estiveram, predominantemente, próximas ou dentro da faixa intermediária de referência (30 a $59 \mathrm{mEq} / \mathrm{L}$ ). Nesse sentido, a relação genótipo-fenótipo na FC, baseada na associação entre as diferentes classes de variantes do gene CFTR e os níveis de cloreto no suor, pode ser uma boa preditora do nível de atividade da proteína CFTR. Pesquisas que observam o efeito de moduladores sobre a função da proteína CFTR têm utilizado os resultados do teste do suor como parâmetros de avaliação da recuperação funcional (ACCURSO et al., 2010; COLLIE et al., 2014).

Apesar da plausibilidade da relação genótipo-fenótipo baseada nas concentrações dos íons cloretos no suor, estas podem ser influenciadas por outros fatores, incluindo a faixa etária dos indivíduos. No presente trabalho, não foi detectada uma correlação entre as concentrações de cloreto no suor e a idade ao diagnóstico. Além disso, foi observada pouca variação nos níveis medianos dos íons de cloro entre as quatro faixas etárias avaliadas. Esses fenômenos podem ter decorrido do pequeno número de indivíduos incluídos e da maior proporção de crianças diagnosticados antes dos dois anos de vida, o que dificulta a comparação entre os grupos etários. O estudo de Traeger et al. (2014) identificou, em indivíduos com FC, níveis de cloreto no suor que diminuíram até o primeiro ano de vida e aumentaram gradualmente nos anos subsequentes, atingindo um pico na meia idade e diminuindo posteriormente. Entretanto, segundo os mesmos autores, as correlações observadas foram fracas e houve o viés de amostragem, já que crianças e jovens foram submetidos em maior proporção ao teste do suor, quando comparados aos adultos e idosos. Por outro lado, Faria et al. (2017) observaram um aumento das concentrações de cloreto no suor até o primeiro ano de idade, seguido de uma gradual redução a partir dos dois anos. Estes últimos autores chamam atenção para o fato de que essas concentrações tendem a ser menores em indivíduos adultos e que existe uma grande variabilidade intra e interindividual.

No que diz respeito ao sexo, as medianas das concentrações de cloreto também mostraram pouca variação entre meninas e meninos. Estes resultados foram semeIhantes àqueles de Cirilli et al. (2018), os quais observaram uma baixa variação entre os níveis de íons de cloro no suor dos indivíduos de diferentes sexos. Os citados autores incluíram em suas análises variáveis como o período do ciclo menstrual. Ismail et al. (2015) evidenciaram a influência do estrógeno sobre a expressão e atividade do canal CFTR em roedores, o que mostra a possibilidade de consideração desse fator na análise dos níveis de cloreto em adolescentes e adultas.

As limitações deste estudo foram constituídas principalmente pela inclusão de um pequeno número de indivíduos de um único centro e pelas características da população analisada, predominantemente pediátrica, cuja maioria concentrou-se em uma única faixa etária. Além disso, a comparação dos níveis de cloreto no suor em três diferentes genótipos identificou um pequeno número de indivíduos no grupo 3.

\section{CONCLUSÃO}

Não houve correlação entre as concentrações de cloreto no suor e a idade dos indivíduos ao diagnostico. Além disso, foi observada uma baixa variabilidade dos níveis iônicos do cloro no suor entre os sexos e as faixas etárias. As diferenças encontradas entre os três grupos de genótipos analisados corroboram a influência das variantes patogênicas sobre a função transportadora da proteína CFTR e o valor preditivo que o teste do suor pode ter na análise dessa função.

\section{REFERÊNCIAS}

ACCURSO, F.J. et al. Effect of VX-770 in persons with cystic fibrosis and the G551D- CFTR mutation. New England Journal of Medicine, Boston, v. 363, n. 21, p. 1991-2003, 2010.

ATHANAZIO, R.A. et al. Diretrizes brasileiras de diagnóstico e tratamento da fibrose cística. Jornal Brasileiro de Pneumologia, Brasília, v. 43, n. 3, p. 219-245, 2017. 
CIRILLI, N. et al. Intra-individual biological variation in sweat chloride concentrations in CF, CFTR dysfunction, and healthy pediatric subjects. Pediatric Pulmonology, New York, v. 53, n. 6, p. 728-734, 2018.

COLLIE, J.T.B. et al. Sixty-five years since the New York heat wave: Advances in sweat testing for cystic fibrosis. Pediatric Pulmonology, New York, v. 49, n. 2, p. 106-117, 2014.

CYSTIC FIBROSIS FOUNDANTION. Clinical and Functional Translation of CFTR (CFTR2). Variant list history. 2020. Disponível em: https://cftr2. org/mutations_history. Acesso em: 18 nov. 2020.

CYSTIC FIBROSIS MUTATIONS DATABASE. CFMDB statistics. 2020. Disponível em: http://www.genet.sickkids.on.ca/StatisticsPage.html. Acesso em: 13 Nov. 2020.

DE BOECK, K.; AMARAL, M. D. Progress in therapies for cystic fibrosis. The Lancet Respiratory Medicine, [s,..], v. 4, n. 8, p. 662-674, 2016.

ELBORN, J.S. Cystic Fibrosis. The Lancet, vLondon, . 388, n.10059, p2519-2531, 2016.

ESPEL, J. C. et al. The relationship between sweat chloride levels and mortality in cystic fibrosis varies by individual genotype. Journal of Cystic Fibrosis, [s.I], v. 17, n. 1, p. 34-42, 2018.

FARIA, A. G. et al. The correlation between age and sweat chloride levels in sweat tests. Revista Portuguesa de Pneumologia, [s.I], v. 23, n. 4, p. 227-230, 2017.

FARRELL, P.M.; KOSCIK, R.E. Sweat chloride concentrations in infants homozygous or heterozygous for F508 cystic fibrosis. Pediatrics, Evanston, v. 97, n. 4, p. 524-528, 1996.

FELDMANN, D. et al. CFTR genotypes in patients with normal or borderline sweat chloride levels. Human Mutation, New York, v. 22, n. 4, p. 340-340, 2003.

FIRMIDA, M.C.; MARQUES, B. L.; DA COSTA, C.H. Fisiopatologia e manifestações clínicas da fibrose cística. Revista Hospital Universitário Pedro Ernesto, [s.I], v. 10, n. 4, 2011.
GIBSON, L.E.; COOKE, R.E. A test for concentration of electrolytes in sweat in cystic fibrosis of the pancreas utilizing pilocarpine by iontophoresis. Pediatrics, Evanston, v. 23, n. 3, p. 545-549, 1959.

ISMAIL, N. et al. Estrogen and progesterone differentially regulate the levels of cystic fibrosis transmembrane regulator (CFTR), adenylate cyclase (AC), and cyclic adenosine mono-phosphate (cAMP) in the rat cervix. Molecular reproduction and development, New York, v. 82, n. 6, p. 463-474, 2015.

MARSON, F.A.L. Disease-modifying genetic factors in cystic fibrosis. Current Opinion in Pulmonary Medicine, [s.I], v. 24, n. 3, p. 296-308, 2018.

PARAD, R.B. et al. Sweat testing infants detected by cystic fibrosis newborn screening. The Journal of Pediatrics, [s.I], v. 147, n. 3, p. S69-S72, 2005.

PEREIRA, S. V. et al. Novel, rare and common pathogenic variants in the CFTR gene screened by high-throughput sequencing technology and predicted by in silico tools. Scientific Reports, [s.I], v. 9, n. 1, p. 1-16, 2019.

SOCIEDADE BRASILEIRA DE PEDIATRIA. Calendário da puericultura. Rio de Janeiro, 2014. Disponível em: https://www.sbp.com.br/fileadmin/ user_upload/pdfs/CalendarioPuericultura_Jan2014.p df. Acesso em: 18 nov. 2020

TRAEGER, N. et al. Relationship between sweat chloride, sodium, and age in clinically obtained samples. Journal of Cystic Fibrosis, Amsterdam, v. 13, n. 1, p. 10-14, 2014.

VIDIGAL, P. V. T. et al. p. F508del in a heterogeneous cystic fibrosis population from Minas Gerais, Brazil. Brazilian Journal of Medical and Biological Research, Ribeirão preto, v. 41, n. 8, p. 643-647, 2008.

WILSCHANSKI, M. et al. Correlation of sweat chloride concentration with classes of the cystic fibrosis transmembrane conductance regulator gene mutations. The Journal of Pediatrics, [s.I], v. 127, n. 5, p. 705-710, 1995.

Submetido em: $07 / 12 / 2020$

Aceito em: 14/12/2020 